\title{
Una investigación sobre la contratransferencia en psicoanálisis
}

\section{A research on counter-transference in psychoanalysis}

\section{Cecilia Greca}

\section{RESUMEN:}

La tesis de Maestría titulada "El concepto de contratransferencia en la obra de Freud y Lacan. Reflexiones sobre su lugar en la teoría y su valor clínico en psicoanálisis" surge del interés por interrogar la notable ausencia del término "contratransferencia" dentro del psicoanálisis orientado por la enseñanza de Lacan. Para ello, se historiza el surgimiento y desarrollo del concepto y se problematizan las lecturas establecidas respecto de la pertinencia del mismo para pensar la teoría y la clínica psicoanalíticas. Se realiza un rastreo del término en la obra de Freud y Lacan, analizando su estatuto y cuestionando las "lecturas consagradas" al respecto. A su vez, se abordan los desarrollos de los autores posfreudianos reconocidos como pioneros en la consideración de la contratransferencia como instrumento en la clínica, así como de los analistas con quienes Lacan polemizó al abordar la cuestión. A partir de esto, se sostiene una interrogación respecto de la validez de desterrar el concepto de la reflexión teórico-clínica del psicoanálisis, en beneficio de conceptos tales como "transferencia" y "deseo del analista".

\section{SuMMARY:}

The Master's thesis entitled “The concept of counter-transference in the work of Freud and Lacan. Reflections on its place in the psychoanalytical theory and its clinical value" is motivated by the interest in interrogating the absence of the term "counter-transference" in psychoanalysis oriented by the teachings of Lacan. For this purpose, it historicizes the appearance and development of the concept and problematizes the established points of view regarding the relevance of this concept in the psychoanalytical theory and clinical practice. The research traces the term in the work of Freud and Lacan, analyzing its status and questioning the "consecrated statements" on the issue. At the same time, the thesis addresses the developments of post-Freudian analysts who are recognized as pioneers in the consideration of counter-transference as an instrument in psychoanalytical treatment, as well as the work of the authors with whom Lacan polemicizes when approaching the matter. As a result, a question is raised regarding the validity of eliminating the concept from theoretical and clinical reflection in 
Palabras Clave:

Psicoanálisis - contratransferencia transferencia - deseo del analista psychoanalysis, replacing it with concepts such as "transference" and "desire of the analyst".

\section{WORD PAD:}

Psychoanalysis - Counter-transference Transference - Desire of the analyst 
ESCRIBIR UNA TESIS SOBRE LA CONTRATRANSFERENCIA

La tesis de Maestría titulada "El concepto de contratransferencia en la obra de Freud y Lacan. Reflexiones sobre su lugar en la teoría y su valor clínico en psicoanálisis" es fruto de una investigación realizada entre los años 2012 y 2016 en el marco de la Maestría en Psicoanálisis que funciona en la Facultad de Psicología de la Universidad Nacional de Rosario. El motivo de la misma fue el interés por interrogar la notable ausencia del término "contratransferencia" dentro del psicoanálisis orientado por la enseñanza de Lacan, historizando el surgimiento y desarrollo del concepto y problematizando las lecturas establecidas respecto de la pertinencia del mismo para pensar la teoría y la clínica psicoanalíticas.

La tesis parte de la idea, planteada por Liliana Baños e Isabel Steinberg (2012), de que el psicoanálisis es una práctica de la dificultad, y como tal, esta es estructural y estructurante. A su vez, podemos afirmar que los mayores obstáculos con los que choca el psicoanálisis son inherentes a su práctica. Si bien son varios los conceptos que atraviesan la reflexión acerca de dichas dificultades, el concepto de contratransferencia constituye el núcleo de la investigación.

Reconociendo la enorme importancia otorgada por Freud, y luego por Lacan, a la transferencia analítica, se destaca el hecho de que la contratransferencia haya sido muy poco trabajada por el padre del psicoanálisis. Por otro lado, luego de una temprana relectura del tema por parte de Lacan se le atribuye el haber desvanecido esta cuestión de la teoría y la reflexión clínica en psicoanálisis, e incluso algunos autores sostienen que la condena de la contratransferencia ha funcionado en el pasado y lo sigue haciendo como marca de pertenencia al psicoanálisis orientado por la enseñanza de Lacan (Miller, 2003:7; Leff, 2007: 65 Cabral, 2009: 25-32). Nos posicionamos críticamente respecto de estos planteos rígidos, reconociendo en la obra del analista francés algunos matices que no permiten cerrar la cuestión en lecturas dicotómicas. Por otro lado, sostenemos que este concepto no puede subsumirse fácilmente en el concepto de "deseo del analista", si bien ambos se encuentran evidentemente entrelazados.

Con el objetivo de realizar un aporte que lleve a profundizar y enriquecer una lectura de la clínica a partir de un deslinde más claro de los conceptos, en la tesis se realiza un rastreo del término en cuestión en la obra de Freud y Lacan, intentando problematizar su estatuto y las "lecturas consagradas" al respecto. Nuestra hipótesis de partida es que la contratransferencia, precisamente por haber llegado a condensar múltiples sentidos que la hacen una noción de mucha densidad histórica y evidentemente problemática, permite pensar e interrogar la complejidad del lugar del analista con una especificidad que no encontramos en conceptos afines.

Así, esta investigación consiste en un trabajo de articulación y profundización conceptual a partir de una elaboración expositivo-argumentativa de la bibliografía específica. Para ello se ha utilizado una metodología que ha sido denominada "metodología de análisis teórico-crítico" (Gentile, 2009: 4), la cual articula dos procedimientos en relativa simultaneidad: el procedimiento constructivo, que implica un trabajo de desarrollo y explicación de los conceptos; y el procedimiento deconstructivo, que implica el trabajo críti- 
co propiamente dicho hacia el interior del tema tratado y también hacia el exterior, es decir, estableciendo líneas de articulación y tensión con otros conceptos del corpus teórico del psicoanálisis.

Por otro lado, se retoman los postulados del paradigma indiciario descripto por Carlo Ginzburg, en el que no se trata de investigar a partir de los grandes rasgos o características más salientes de un objeto o una situación, sino de "examinar los detalles más omitibles” (Ginzburg, 2004: $70)$, los “indicios que a la mayoría le resultan imperceptibles” (Ginzburg, 2004: 72 ), con el fin de llegar a una verdad que hasta el momento permanecía oculta. Dado el carácter elusivo del concepto de contratransferencia, realizar una lectura inspirada en este paradigma, que retome la tradición misma del psicoanálisis como método, resulta una tarea ineludible. Más allá de las menciones explícitas de la cuestión, en la tesis se buscan indicios que permitan ubicar a la contratransferencia en los escritos freudianos y lacanianos, fundamentalmente, para construir a partir de éstos una multiplicidad de puntos de vista que permitan dar al concepto toda su densidad teórico-clínica.

Además, al tratarse de una tesis que toma como tema central un concepto psicoanalítico eminentemente problemático, consideramos pertinente dar a esta investigación una inspiración ensayística. Con esto hacemos referencia al recurso al ensayo como forma, tal como lo ha caracterizado Theodor Adorno (1962). Retomamos de este autor la idea del ensayo como un medio para dar lugar a la interacción de los conceptos, sin pretender una definición acabada de los mismos e impugnando así un abordaje lineal de las cosas. En esta línea tomamos también los aportes de Alberto Giordano, quien, haciendo referencia al recurso al ensayo, devuelve su dignidad al "asombro, el desconcierto y el vértigo de la conjetura que experimentan los sujetos en trance de saber" (Giordano, 1998: 55). Se trata, así, de restituir el vínculo entre los conceptos y los problemas e interrogantes que les dieron origen y los siguen atravesando, a través de una lectura crítica y una escritura problematizante.

Como punto de partida para interpelar la idea del borramiento de la contratransferencia en el psicoanálisis lacaniano, la tesis ubica la actualidad del debate en torno a la misma. Para ello, partimos de la discusión que parece haber reactualizado en el medio psicoanalítico dicho debate: un intercambio sostenido en el año 2002 entre Daniel Widlöcher -en aquel entonces presidente de la Asociación Psicoanalítica Internacional (IPA)- y Jacques-Alain Miller -fundador y en aquel momento presidente de la Asociación Mundial de Psicoanálisis (AMP)-, titulado "El porvenir del psicoanálisis”. Ese nombre resulta significativo por su similitud con el título de la ponencia en la que Freud introduce en 1910 el concepto de contratransferencia en la comunidad analítica: "Las perspectivas futuras de la terapia psicoanalítica”.

En el marco de este encuentro, Widlöcher ubica la centralidad que tiene, para los analistas de la IPA, la asociación libre y la atención flotante, en el marco de una relación que tiene dimensiones transferenciales y contratransferenciales. Esto define, para él, la escucha analítica. Por su parte, Miller encuentra en la contratransferencia la posibilidad de dar entidad a la división entre "la práctica que procede de Lacan" y "todas las demás" (Widlöcher, Miller y Granger, 2002: 1059) y denuncia, en los analistas que han dado lugar 
a la contratransferencia en sus reflexiones teórico-clínicas, un excesivo interés puesto en los procesos internos del propio analista. Esto está en consonancia con las ideas transmitidas por Miller en el curso dictado en el marco del Departamento de Psicoanálisis de Paris VIII, durante los primeros meses del año 2002, es decir, poco antes del debate con Widlöcher. Allí dice a su auditorio que podemos ver a la enseñanza de Lacan "como un rechazo de la contratransferencia, modulado incesantemente de formas diversas" (Miller, 2003: 7). Respecto de esto, seguimos a Alberto Cabral (2009: 25-32) cuando se posiciona críticamente respecto de las mencionadas declaraciones y plantea que esto lleva a darle a la noción el estatuto de un "emblema" que resulta en que se sustrae a la contratransferencia su densidad conceptual y los matices que conlleva.

Como resonancias de esta discusión, en el seno de instituciones tan diferentes como la Escuela de Orientación Lacaniana (EOL, adherida a la AMP), la Escuela Lacaniana de Psicoanálisis (ELP), la Sociedad de Psicoanálisis Freudiano (SPF) y la Asociación Psicoanalítica Argentina (APA, adherida a la IPA), surgieron artículos, revistas e incluso libros enteros dedicados a la cuestión. La tesis rastrea y comenta dichas publicaciones, centrándose en aquellas en las que los autores se reconocen como tributarios de la enseñanza de Lacan. A su vez, en su selección se intenta reflejar la diversidad de perspectivas y lecturas que coexisten bajo dicho reconocimiento, tomando como referencia las diversas pertenencias institucionales de los autores (si bien reconocemos que no se trata de un criterio exhaustivo). A partir de esto, se articulan interrogantes que serán retomados a lo largo de la investiga- ción: ¿es posible pensar en un sujeto (si no rehusamos que quien está en posición de analista es, a fin de cuentas, un sujeto) que pueda desprenderse a tal punto de su condición de tal que lleve adelante una escucha despojada de toda singularidad? ¿Es efectivamente posible escuchar el discurso de un sujeto y realizar intervenciones, sin que esto implique algún tipo de actividad de pensamiento? ¿Es esto lo que implica el "deseo del analista" como condición para ejercer el análisis? ¿La participación del analista en la cura se reduce a la puesta en juego de dicha función? ¿La apelación a la contratransferencia como concepto implica necesariamente la encerrona imaginaria en el análisis? Y en esta línea, ¿el único estatuto posible para la contratransferencia es el de ser objeto de eliminación o de fascinación del analista?

La tesis se estructura en tres partes que ordenan el recorrido. En la primera parte se toma como punto de partida la conceptualización de la transferencia en la obra freudiana, para a partir de allí llevar adelante un rastreo del concepto de "contratransferencia". En el texto se destacan los diferentes puntos de la obra en que podemos ubicar el concepto tanto de manera explícita como implícita, interrogándose acerca de los matices y alcances del mismo. Asimismo, se aborda la cuestión no sólo en los textos teóricos del autor, sino también en la lectura de algunos de sus casos clínicos más importantes. Finalmente, para poner en perspectiva la concepción freudiana de la contratransferencia, se dedica un apartado a los trabajos de Sándor Ferenczi que abordan el tema, dado que se trató de un importante interlocutor y discípulo de Freud, y ya que es precisamente respecto de este tema que podemos pensar el alejamiento de ambos. 
Seguidamente, con el objetivo de abordar las conceptualizaciones lacanianas en toda su riqueza, la tesis realiza un recorrido por los postulados de autores posfreudianos que han hecho valiosos aportes en relación al tema. En este sentido, la investigación se centra en aquellos con los que polemiza Lacan en el camino que lo llevó a repensar la cuestión de la contratransferencia, ubicando como eje los seminarios "La transferencia" (1960-1961) y “La angustia” (1962-1963), en los que aborda específicamente esta temática. Por otro lado, se da un lugar a los autores que la bibliografía consultada ubica como pioneros respecto de esta temática (Etchegoyen, 1986; de León y Bernardi, 2000; Miller, 2003; Widlöcher, 2011), ya que si bien no son el eje de los comentarios de Lacan, se observan marcas de su influencia en la letra de aquellos psicoanalistas que más tarde fueron objeto de los comentarios más célebres del psicoanalista francés. De esta manera, en un primer momento se estudian los aportes de Donald Winnicott y Paula Heimann. Por otro lado, y por su importancia en el contexto psicoanalítico argentino, se realiza un comentario de los aportes de Heinrich Racker, quien fuera un importante exponente de la formación y la investigación en la APA, ya que se trata de alguien que representa cómo la cuestión de la contratransferencia se puso en juego en el contexto institucional de nuestro país. Horacio Etchegoyen (1986: 241 y sigs.), en el estudio que realiza de los aportes principales que se hicieron desde la época de Freud a la cuestión de la contratransferencia, ubica a la teoría de Racker a la altura de los aportes de Heimann, ya que siendo simultáneos y sin embargo autónomos, tienen el valor de ser pioneros en la elaboración de teorías originales so- bre el tema. Con este recorrido como base, posteriormente se estudian los planteos de Roger Money-Kyrle, Margaret Little y Lucy Tower.

En la tercera parte de la tesis se trabajan los desarrollos que Lacan ha hecho al respecto, para lo cual se realiza un recorrido por los escritos y seminarios pertinentes, intentando ampliar la lectura que clásicamente se ha hecho de la conceptualización lacaniana de la contratransferencia. A partir de esto, se sostiene una interrogación respecto de la validez de desterrar el concepto de la reflexión teórico-clínica del psicoanálisis, en beneficio de conceptos tales como "transferencia" y "deseo del analista”.

\section{LA CONTRATRANSFERENCIA EN FREUD: RECONOCERLA Y DOMINARLA}

A fin de abordar la manera en que Freud introduce la cuestión de la contratransferencia en su obra, la tesis realiza primero un recorrido por los textos en los que el padre del psicoanálisis desarrolla su concepción de la transferencia analítica. A su vez, se toman tres de los casos paradigmáticos de Freud -el caso del hombre de las ratas, el caso Dora y el caso de la joven homosexual- como base para desarrollar los dos roles posibles que Freud asigna a la transferencia en el análisis: "por un lado, un recurso auxiliar de valor insustituible; por el otro, una fuente de serios peligros" (Freud, 1940 [1938]: 175). En esta línea, la lectura del caso Dora y de la joven homosexual, acompañada por la interpretación de Lacan en "Intervención sobre la transferencia” (1951), nos permite una vía de acceso a las "trampas" de la contratransferencia. 
Por su parte, el término hace su primera aparición pública en el año 1910, en el discurso inaugural del segundo Congreso Internacional de Psicoanálisis que fue titulado "Las perspectivas futuras de la terapia psicoanalítica”. No obstante, el tema ya era objeto explícito de debate en la correspondencia de Freud con sus discípulos, y en particular, con Jung a propósito de su relación con Sabina Spielrein (Leff, 2007: 113, n.12; Guyomard, 2011: 21-24; Weissmann, 1994: 564-570; Geissmann, 2002: 300). De hecho, la primera mención del término data de 1909 y tiene lugar en una carta dirigida a su discípulo. En este punto, es significativa una apreciación hecha allí por el padre del psicoanálisis, que contrasta de manera significativa con su posición pública al respecto. Freud le dice a Jung que la contratransferencia, lejos de provocar un perjuicio, brinda al analista el recurso de "endurecerle la piel" ya que se trata de una cuestión permanentemente presente que él debe aprender a manejar. Así, la caracteriza como una "bendición disfrazada" [a blessing in disguise], ya que enseña mucho al analista (en Weissmann, 1994: 565). Ahora bien, nunca vacila respecto de la necesidad de controlar estos fenómenos y la dificultad propia de esta tarea, y esta posición se refuerza en sus textos publicados. Freud siempre sostuvo la necesidad de sofrenar la contratransferencia, y articuló esta noción con la idea de la abstinencia. En este recorrido, se destacan las metáforas utilizadas por Freud para dar cuenta de la posición del analista: el analista como cirujano, como espejo (Freud, 1912: 114, 117) y como químico (1915 [1914]: 173). Lejos de la insensibilidad y la opacidad que implican los primeros dos términos de comparación, la metáfora del químico da lugar a "las fuer- zas más explosivas” (1915 [1914]: 173) y a las implicancias de su manipulación por parte del analista. De este modo, en palabras de Patrick Guyomard, "le da lugar al imprevisto, a la resistencia de la materia, a la participación de cada uno en tanto que elementos en presencia. Reacciones imprevistas, explosiones, torpezas y errores de manipulación están contemplados [sont au programme]" (Guyomard, 2011: 25). Si bien el término desaparece de la obra escrita en 1915, los obstáculos del análisis y los "puntos ciegos" del analista siguieron ocupando a Freud hasta el final de su obra.

Atendiendo entonces a la posibilidad de rastrear el concepto en lugares en donde no es objeto explícito del abordaje freudiano, la tesis sigue la idea propuesta por Steinberg (en Baños y Steinberg, 2012: 98-99), quien plantea la posibilidad de reconstruir el concepto de contratransferencia a partir de los textos en los que Freud aborda los llamados "fenómenos telepáticos". Este apartado de la primera parte se centra particularmente en el caso del "Señor P." que Freud expone en la la $30^{\mathrm{a}}$ conferencia de las "Nuevas conferencias de introducción al psicoanálisis", titulada "Sueño y ocultismo" (1933 [1932]), en el que se aborda un aparente fenómeno de transmisión de pensamiento en una sesión analítica. Luego de un estudio pormenorizado de dicho caso y de las reflexiones de Freud al respecto, se establece la hipótesis de que la posición tajante de éste respecto de la contratransferencia lleva en ocasiones a su pleno desconocimiento y obtura la posibilidad de leerla, llevando a concebir sus efectos como una "experiencia extrasensorial”. Es lo que Jaime Fernández Miranda (2016) denomina "renegación freudiana de la contratransferencia". 
Finalmente, este momento de la tesis culmina con un abordaje de la obra de Ferenczi, ubicando sus deslizamientos respecto de la técnica analítica, desde una posición más cercana a Freud en la década de 1910 hasta su propuesta del análisis mutuo en la década de 1930. Allí también se reflexiona sobre los efectos del análisis de Ferenczi con su maestro, y de la posición paterna asumida por este en la transferencia, en las teorizaciones del discípulo. Se destaca el énfasis puesto por Ferenczi en la comprensión del analista, un punto que estará muy presente en todos los autores posfreudianos dedicados al tema de la contratransferencia. Por otro lado, es interesante que para este discípulo de Freud las emociones y disposiciones internas del analista sean la condición misma de esta comprensión: el comprender depende de "sentir con" y "sentir por" el otro. Fernández Miranda sostiene así que "Ferenczi devendrá el principal teórico del analista, haciendo ingresar, por así decir, al analista en el análisis” (2016). Por otro lado, se destaca la importancia atribuida por el autor al hecho de sostener la tensión entre la afectación del analista a partir del discurso del paciente -que está en la base de la idea de que los actos psíquicos del analista pueden decir algo del psiquismo del analizante- y el reconocimiento de la alteridad de este.

Al finalizar esta parte de la tesis quedan planteados dos interrogantes: ¿reconocer el modo en que la palabra del analizante afecta al analista, dar un lugar a la contratransferencia en la reflexión clínica, lleva necesariamente a la simetría, a la confusión de lugares, a una especie de "análisis mutuo" al estilo de Ferenczi?; y en segundo término, ¿resulta válido limitar la cuestión de la contratransferencia a la supuesta insuficiencia del análisis personal del analista?

\section{LA CONTRATRANSFERENCIA EN EL CENTRO DEL DEBATE. \\ LOS ANALISTAS POSFREUDIANOS.}

La firmeza en los planteos de Freud y la innegable influencia de Ferenczi en generaciones sucesivas de analistas nos llevan a una época posterior del psicoanálisis, en que el silencio acerca de la contratransferencia comenzó a ser cuestionado. Hasta mediados de la década de 1940, la posición freudiana respecto de la contratransferencia, esto es, la necesidad de reconocerla y dominarla, era la mayoritaria dentro de los círculos analíticos. Es hacia fines de esa década y fundamentalmente durante los años '50 que esta posición fue sometida a revisión. Comenzaron a aparecer críticas al "analista espejo", el "ideal del analista "distanciado»" (Heimann, 1949-1950: 43) y el "analista impersonal" (Little, 1951), reivindicando el lugar de la contratransferencia como instrumento esencial en la dirección de la cura. Widlöcher destaca que “(...) es sobre todo de Londres que vinieron dos corrientes paralelas que pusieron el acento en el interés de la implicación afectiva del analista en la comprensión del proceso de la cura y por lo tanto en el manejo de la escucha interpretativa” (Widlöcher, 2011: 96). Estas dos corrientes fueron las inauguradas por Donald Winnicott y Paula Heimann. Paralelamente, Heinrich Racker en Argentina producía un giro similar en la cuestión. A partir de entonces proliferaron los artículos psicoanalíticos respecto del tema, en los que diferentes autores asumían posiciones disímiles al respecto. En 
este sentido resulta interesante la lectura de Etchegoyen (1986), quien afirma que el verdadero cambio no tuvo que ver con aceptar el carácter ineludible de la contratransferencia en el análisis, sino en darle el estatuto de un instrumento (importante; necesario; fundamental; dependiendo del autor de referencia) para la comprensión del paciente.

Luego de un recorrido por los planteos de Winnicott, Heimann y Racker, la tesis aborda los postulados de los tres analistas con quienes Lacan discute en el marco de su seminario, entre 1960 y 1963: Roger Money-Kyrle, Margaret Little y Lucy Tower.

Dentro de la posición de Money-Kyrle, se destaca su conceptualización de la "contratransferencia normal", caracterizada por la existencia de "satisfacciones reparatorias" y de cierta "disposición paternal" del analista (Money-Kyrle, 1956: 151). Esto permite la "empatía” hacia el paciente (que tiene como base una identificación parcial con éste) que permite comprenderlo, más allá del conocimiento teórico de quien dirige la cura. A su vez, Money-Kyrle afirma que los momentos en que los sentimientos del analista se encuentran perturbados son ineludibles y "es precisamente en ellos que el analista, analizando silenciosamente sus propias reacciones, puede aumentar su "insight", disminuir sus dificultades, y aprender más acerca de su paciente" (Money-Kyrle, 1956: 163). En este sentido, el autor sostiene que la contratransferencia tiene no sólo efectos, sino también sus causas en el paciente. Esto lleva a la importancia de poder vislumbrar de qué manera éste contribuyó a crear el conflicto presente en el analista, como requisito para la instrumentación adecuada de los fenómenos contratransferenciales a los fines de la comprensión del psiquismo del paciente.

$\mathrm{Al}$ abordar los aportes de Margaret Little, la tesis articula sus desarrollos teóricos con reflexiones respecto de los efectos de los análisis de la autora conducidos por Ella Sharpe y luego por Donald Winnicott. Para ello se recorren fragmentos del relato de análisis realizado por la propia Little. Se plantea que es en la tensión entre estos dos análisis que pueden entenderse las consideraciones de la autora respecto de este tema; fundamentalmente, su insistencia en la comunicación de la contratransferencia al paciente, en el derecho de éste de conocer dichos procesos internos de su analista, y su descripción de "la respuesta total del analista" (Little, 1957). A su vez, se realiza un abordaje del caso Frida (Little, 1957), sobre el cual Lacan articulará posteriormente algunas reflexiones críticas.

Finalmente, esta segunda parte de la investigación aborda extensamente el artículo de Lucy Tower titulado "La contratransferencia" (1955), sobre el que Lacan centrará sus reflexiones sobre la temática en su seminario "La Angustia". En dicho artículo la autora afirma que aunque la contratransferencia está enraizada en estructuras neuróticas del analista, el surgimiento de ciertas manifestaciones en un análisis en particular puede servir de orientación respecto de ese analizante. En esta misma línea, afirma que la contratransferencia tiene el papel de un catalizador en el proceso terapéutico, es decir, su comprensión estimula y acelera el trabajo del analista en la intelección y resolución de la neurosis del paciente. Esto es así ya que se trata de una "comprensión emocional" por parte del analista (Tower, 1955: 122), que no se confunde con la compren- 
sión intelectual de los procesos psíquicos en juego, sino que va más allá, siendo condición del "verdadero insight" (Tower, 1955: 125). No obstante esto, el valor de orientación para el analista que tienen los fenómenos contratransferenciales en la concepción de Tower no significa que éstos deban ser compartidos con el paciente, posición que se distancia de los postulados de Little. Luego de establecer las ideas centrales de la autora respecto del papel de la contratransferencia en el análisis, la tesis recorre los cuatro casos clínicos presentados en el escrito, que luego serán retomados al abordar el tratamiento que hace Lacan de este texto.

\section{LACAN O LA “CRÍTICA DE LA CONTRATRANSFERENCIA"}

La tercera parte de la tesis comienza haciendo referencia al llamado "retorno a Freud" que promueve Lacan al comienzo de su enseñanza, enfatizando los efectos del mismo respecto de una transformación del psicoanálisis ya que se realiza una rearticulación de los planteos freudianos gracias a nuevas coordenadas conceptuales. La tesis se refiere específicamente al concepto de transferencia y a las implicancias de pensarla a partir de los tres registros: simbólico, imaginario y real. Esto tiene profundos efectos en la manera de pensar el análisis y el lugar del analista allí: queda establecido que la concepción de la transferencia no puede quedar reducida al plano dual, especular, imaginario, para lo cual Lacan introduce a la palabra como tercer término necesario para aprehenderla. Al plantear, en articulación con esto, la noción de gran Otro -consustancial al registro simbólico- se afirma la idea de una alteridad radical más allá de la alteridad especular, con lo cual se deshace la ilusión de reflexividad, de conocimiento recíproco. En este primer momento de su enseñanza, el lugar del Otro le aporta a Lacan una clave para pensar el lugar que el analista debe venir a ocupar a fin de que progrese el análisis, así como para despejar sus posibles impasses. En este punto se destaca la lectura lacaniana de los casos de Dora y la joven homosexual y su interpretación de por qué se detuvo en ellos la dialéctica analítica.

Ahora bien, el Otro no es absoluto; esto es lo que Lacan designa como A barrado y que articula cuando dice que "no hay Otro del Otro", lo que significa que no hay en el Otro ningún significante que pueda oportunamente responder por lo que soy. Se retoman las palabras de Juan Ritvo cuando dice que "en el Otro algo no responde, literalmente: no responde. ¿A qué? A la pregunta por el último secreto de mi nombre en tanto sujeto deseante. Allí, en el Otro no hay ninguna respuesta. En el Otro, lo único que hay es un vacío, o más precisamente, un agujero" (Ritvo, 1997: 72). Esto en un principio es articulado a partir de postular que el falo como significante, $\Phi$ [phi mayúscula], como signo del deseo, es el significante que designa la falta de significante (Lacan, 19601961: 264). Hasta este momento, Lacan articulaba la cuestión de la falta a nivel del significante, e instituía al falo, en su dimensión simbólica, como el significante de la falta $(\Phi)$. En ese momento de su enseñanza se trataba del a como objeto del deseo, como señuelo. La novedad que se introduce en el seminario "La Angustia” es que habría una falta irreductible al significante, una falta radical, "radical en 
la constitución misma de la subjetividad" (Lacan, 1962-1963: 148).

De este modo, el objeto $a$ se constituye como resto en la relación del sujeto con el Otro, y en cuanto tal resiste a toda asimilación a la función del significante. "Pero precisamente este desecho, esta caída, esto que resiste a la significantización, es lo que acaba constituyendo el fundamento en cuanto tal del sujeto deseante" (Lacan, 1962-1963: 190). En la respuesta del Otro a la pregunta del sujeto acerca de sí mismo, queda el enigma; hay en el Otro una falta que no completa el deseo de saber del sujeto. Éste va a constituirse entonces como sujeto barrado en la medida en que se hace cargo de esa falta en el Otro, de manera inconsciente. De esa operación queda un resto, $a$, objeto causa de deseo. Esto va a permitir pensar el lugar del analista a otro nivel y nuevamente permite leer los impasses freudianos bajo una nueva luz: la no articulación de la diferencia entre el objeto causa de deseo y el objeto parcial como señuelo conlleva que la posición del analista quede coagulada en este último lugar.

Por otro lado, la puesta a punto del estatuto del objeto a como causa de deseo es lo que le permitirá a Lacan dar razón de lo que designa bajo el nombre "deseo del analista". Ya en el seminario "La transferencia" se hablaba de la posición del analista y de qué podía significar hablar del "deseo del analista". Lacan es muy claro al afirmar que "la complejidad de la cuestión de la transferencia no se podía en absoluto limitar a lo que ocurre en el sujeto llamado paciente, en el analizado. En consecuencia, se plantea la cuestión de articular (...) qué debe ser el deseo del analista" (Lacan, 1960-1961: 124). Tomando como ejemplo la posición de
Sócrates en "El Banquete", Lacan afirma que se trata para el analista de ocupar el lugar de "deseante puro" (Lacan, 19601961: 410). El analista debe ser capaz de sostener el deseo en cuanto tal, sin obturar con postizos el deseo del sujeto. El lugar del objeto a minúscula (recordemos que aquí todavía se trata del objeto en el fantasma) “el analista, por su parte, sólo puede pensar que cualquier objeto puede rellenarlo (...) No hay objeto que valga más que otro - éste es el duelo a cuyo alrededor se centra el deseo del analista" (Lacan, 1960-1961: 440). Ahora bien, tal como afirmamos anteriormente, introducir el objeto $a$ en su estatuto real por fuerza vendrá a modificar la concepción del objeto en el deseo, y por consiguiente en el deseo del analista. Lacan afirma que el deseo del analista, como todo deseo, tiene una referencia interna al objeto $a$, causa de deseo. Se trata de que quien ocupe el lugar de analista sea alguien que "por poco que sea, por algún lado, algún borde, haya hecho volver a entrar su deseo en este $a$ irreductible" (Lacan, 1962-1963: 365). De esta forma, el análisis puede ser llevado más allá de la demanda y de la identificación, al plano del deseo y al encuentro del sujeto "con la porquería que le sirve de soporte, el objeto $a$, cuya presencia, puede decirse legítimamente, es necesaria" (Lacan, 1964: 266).

Así, luego de sostener durante años que el analista debía ocupar el lugar del Otro en la relación analítica, para no caer en los impasses de un psicoanálisis reducido al registro imaginario, Lacan debió introducir en la maniobra y la operación de la transferencia la incidencia del objeto a. Así, el analista, gracias a su análisis personal, debe poder servir de soporte al objeto $a$, ser un Otro en tanto que no es 
absoluto, si quiere ir más allá de una identificación idealizante que obture la falta radical constitutiva del deseo. Se trata de poner en juego el $a$ en tanto falta irreductible al significante y de que el analista se sitúe como A barrado para que el análisis no quede detenido en la impotencia, en el $-\varphi[$ el impasse freudiano, tal como lo sitúa Lacan]. En este marco es que se plantea la pregunta acerca de la pertinencia del concepto de contratransferencia en psicoanálisis.

La tesis sitúa como punto de partida para abordar los planteos de Lacan respecto de la contratransferencia la definición de la misma que el autor postula en 1951 y que se ha hecho célebre: "la suma de los prejuicios, de las pasiones, de las dificultades, incluso de la insuficiente información del analista en determinado momento del proceso dialéctico" (Lacan, 1951: 219). Cuestionando la idea de que la posición lacaniana respecto de este tema pueda cerrarse allí, la investigación recorre las menciones que el analista francés realiza de la cuestión a lo largo de seminarios y escritos. Esta queda ubicada en el registro imaginario, en el terreno de la relación de yo a yo; también es definida como los sentimientos del analista, respecto de los cuales Lacan dice que "sólo tienen un lugar posible en este juego, el del muerto; y que si se lo reanima, el juego se prosigue sin que se sepa quién lo conduce” (Lacan, 1958: 563). En este punto la tesis toma la caracterización que realiza Gloria Leff de la función del "dummy" o "muerto" en el bridge, para cuestionar la idea de rigidez y cadaverización que evoca el término "muerto", en favor de la idea de que se trata de un jugador "que abre sus cartas para que otro juegue con ellas (...) Pero no puede intervenir, es dirigido por su partenaire" (Leff, 2007: 121, $n$. 7). Por otro lado, el planteo de que "en el otro con minúscula que hay en él [se refiere al analista] tiene que haber algo capaz de jugar al muerto" (Lacan, 1960-1961: 216) nos conduce a la idea de un artificio, dejando abierta la pregunta acerca de otro destino posible para la contratransferencia, diferente de su proscripción.

A continuación la tesis se centra en los planteos de los seminarios "La transferencia" (1960-1961) y "La Angustia" (1962-1963), en los que Lacan aborda específicamente la cuestión de la contratransferencia. En uno y otro caso, para articular sus propias elaboraciones toma textos de reconocidos psicoanalistas posfreudianos que se dedicaron al tema para someterlos a una lectura crítica. Reencontramos aquí a Money-Kyrle, Little y Tower. En el marco de estos comentarios Lacan realiza una fuerte crítica respecto de la idea, generalizada entre los posfreudianos, de que un analista debe "comprender" y sostiene que "lo más importante de comprender en la demanda del analizado es lo que está más allá de esta demanda. El margen del deseo es el de lo incomprensible. En la medida en que todo esto no es percibido, un análisis se cierra prematuramente y, por decirlo todo, está malogrado" (Lacan, 1960-1961: 239).

En este momento de su enseñanza, al abordar la cuestión de la transferencia del lado del analista, Lacan entenderá por “contratransferencia”, la "implicación necesaria del analista en la situación de transferencia, y por eso precisamente debemos desconfiar de ese término impropio" (Lacan, 1960-1961: 227). Cabral retoma esta idea de la "impropiedad" y la diferencia de otras traducciones que hablan de la contratransferencia como una "mala pal- 
abra” (Lacan, 1958: 559), criticando así una división de los conceptos entre "buenos" y "malos" que ha contribuido al efecto de borramiento de la contratransferencia en el psicoanálisis lacaniano. Por el contrario, la idea de "impropiedad", con lo que implica de incomodidad e inadecuación, llama a la reflexión crítica y a complejizar la concepción del lugar del analista.

Ahora bien, es en el seminario "La Angustia", momento en el que Lacan ubica al objeto a como objeto causa de deseo, que Leff encuentra la posibilidad de leer una posición novedosa de Lacan respecto de este tema. Siguiendo a la autora, la diferencia entre un manejo exitoso de la contratransferencia y un radical fracaso consiste en cómo el analista se posiciona respecto del objeto $a$ : puede comportarse como quien contiene realmente el objeto que el paciente busca en él, o puede "jugar el papel” (Leff, 2007: 222) sin dejar de advertir que se trata de algo más allá de su persona. Esto permite, a nivel teórico, establecer la diferencia entre operar a nivel del objeto parcial falicizado $(\varphi)$ -límite encontrado por Freud- y el objeto a como causa. En este punto se pone en primer plano la diferencia sustancial entre el deseo del analista y el deseo de la persona que ocupa ese lugar, sostenido en su fantasma. El deseo del analista como función es lo que permite que el lugar de la causa no se llene, que el enigma funcione, que no se caiga en la tentación de llenar el agujero causal con sentido.

Refiriéndose a los autores posfreudianos, afirma que "en la dificultad del abordaje de estos autores en lo referente a la contratransferencia, lo que constituye el obstáculo es el problema del deseo del analista” (Lacan, 1962-1963: 163). Se sostiene que no hay nada en esta frase que pueda llevarnos a pensar en una sustitución de términos, sino muy por el contrario, nos presenta al deseo del analista como una función necesaria que estaría en la base de toda posibilidad de posicionarse de manera correcta respecto de la contratransferencia. En este sentido, podemos establecer una diferencia de niveles en lo que dice Lacan cuando afirma que "El término contratransferencia apunta a grandes rasgos a la participación del analista. Pero más esencial es el compromiso del analista (...)" (Lacan, 1962-1963: 163). Siguiendo las reflexiones de Lacan en este momento de su enseñanza, se propone la idea de que la función "deseo del analista" es la que designa el carácter de ese compromiso del analista, lo cual es del todo esencial en su posibilidad de posicionarse en la relación transferencial, de "participar" en la transferencia (sentido que le otorga, como ya hemos visto, a la contratransferencia). De ahí que Lacan afirme que la cuestión de la contratransferencia no es realmente el problema, mientras no esté resuelta cabalmente la cuestión del deseo del analista.

Es gracias a un análisis de los casos clínicos presentados por Tower que Lacan logra dar un paso más. Refiriéndose a los desarrollos de la analista americana, afirma que "si hay algunas personas que han dicho sobre la supuesta contratransferencia algo sensato, son únicamente mujeres" (Lacan, 1962-1963: 168). En la tesis se interroga la expresión "supuesta contratransferencia", encontrando allí una articulación con la noción de "deseo del analista". A propósito de las mujeres y la contratransferencia, Lacan sostiene que "si se mueven más cómodamente en sus escritos teóricos, es, presumo, porque tampoco se mueven mal en la práctica, 
aunque no vean $-\mathrm{O}$ mejor dicho, no articulen (...)- su mecanismo de un modo del todo claro" (Lacan, 1962-1963: 193). Ya que a continuación Lacan afirma que la mujer sabe muy bien lo que es el deseo del analista, podemos pensar, a partir de estos desarrollos, que esta sería precisamente la función que permitiría articular el mecanismo de la contratransferencia. En este punto, la tesis realiza un seguimiento pormenorizado del análisis de Leff de los dichos de Lacan al leer los casos de Tower, lo cual permite llegar a las dos posiciones respecto del deseo desarrolladas en el seminario: la "masculina" -que pasa por la negativización del falo- y la "femenina" -en la cual "con lo que se enfrenta es precisamente con el deseo del Otro en cuanto tal, y ello tanto más cuanto que, en esta confrontación, el objeto fálico sólo interviene para la mujer en segundo lugar y en la medida en que desempeña un papel en el deseo del Otro" (Lacan, 1962-1963: 200).

En este contexto, se propone pensar la contratransferencia como referida al posicionamiento del analista respecto del objeto $a$, abriéndose así la posibilidad de no quedar coagulado en la posición de ser la sede del objeto parcial. De este modo, Leff (2007: 241) afirma que se abren dos alternativas y dos destinos posibles para el análisis: o bien el analista se posiciona como sede del objeto parcial y es inaccesible eróticamente -lo que lleva a un análisis interminable-, o bien el objeto $a$ yace en el espacio analítico, lo cual permite que se ponga en juego en el analizante como causa de deseo. En esta segunda posición, el análisis puede llevarse más allá de la angustia de castración. La autora afirma que aquí encontramos la formulación lacaniana de la contratransferencia a partir de la invención del objeto $a$ : "el analista en posición de partenaire femenino" (Leff, 2007: 241), lo cual le permite prestarse a cumplir el papel asignado en la transferencia, sostener la mascarada, sin por ello quedar capturado allí.

Si bien en la tesis no se adhiere a los planteos de Guyomard (2011: 188) respecto de que Lacan le dio a la contratransferencia el estatuto de un concepto, y se reconoce la extensa crítica de éste a dicho término fundamentalmente por la carga de sentido resultante del tratamiento recibido a lo largo de las décadas, se destaca que por su misma "impropiedad" la contratransferencia permitió a Lacan una fecunda interrogación de la clínica y de la posición del analista.

\section{¿UN ASUNTO SUPERADO O UN ASUNTO CLAUSURADO?}

En el capítulo final, la tesis se abre a una interrogación por el lugar del concepto de contratransferencia en la elaboración psicoanalítica actual. Se formula la pregunta respecto de si se trata de un asunto que ha sido superado en el transcurso de la historia del psicoanálisis, un concepto que ha sido reemplazado por otros más "pertinentes" y "valiosos" para la reflexión clínica; o si por el contrario cierto "efecto de escuela" ha significado la clausura de una interrogación que pone en el centro de la escena al analista y su implicación y posicionamiento en el análisis. Con el objetivo de no cerrar prematuramente el interrogante, se comparte la postura de Winnicott cuando afirma que "será fútil cualquier discusión que se base en las fallas del análisis del propio analista. En cierto sentido, esto pone fin al debate" 
(Winnicott, 1960: 207-8). Por otro lado, se destaca la pluralidad de puntos de vista dentro del psicoanálisis, buscando evitar su compartimentación en "sectores” pretendidamente homogéneos.

Luego de un recorrido por la obra freudiana y los planteos de los analistas posfreudianos con quienes Lacan polemiza al abordar la cuestión de la contratransferencia, la investigación se introduce en las ideas de Lacan sobre el tema, reconociendo desde el inicio la diversidad de lecturas existentes y la complejidad de la problemática. Se plantea que si bien la contratransferencia no tiene en este nuevo contexto teórico un estatuto conceptual delimitado, la posición del analista francés es mucho más difícil de ceñir en una lectura única de lo que suele decirse al evocar su enseñanza. Por otro lado, el abordaje de otros autores permite abrir preguntas en los intersticios de la reflexión lacaniana.

La introducción del "deseo del analista” para dar cuenta del lugar de quien conduce la cura lleva a repensar la función del análisis personal y el significado de poner entre paréntesis el yo del analista. A partir de este recorrido, se considera que funcionar como soporte del objeto $a$, tal como lo concibió Lacan, no significa dejar de ser un sujeto, pero sí permitir que el único sujeto en análisis sea el analizante. Ahora bien, este trabajo de investigación nos llevó a distinguir entre el deseo del analista, que se refiere al compromiso de éste, a la función que sostiene su lugar, y la contratransferencia, término complejo en el que se han condensado discusiones y posiciones muy diferentes con el correr de las décadas. A partir de recorrer las diversas caracterizaciones de Lacan, encontramos que podemos entenderla como la implicación del analista en la transferencia, su participación en ella, y siguiendo a Leff, planteamos que su manejo depende del posicionamiento del analista respecto del objeto $a$, lo cual vuelve a llevarnos al concepto de deseo del analista y lo que este habilita. Así, acordamos con la idea de que no es en la contratransferencia que encontraremos la posibilidad de designar la especificidad de la función del analista en la cura, y que en este sentido el deseo del analista nos aporta más elementos para pensarla. No obstante, esto no significa desconocer el enorme valor que ha tenido la contratransferencia en la posibilidad de articular una rigurosa interrogación respecto de la práctica analítica.

La lectura de los posfreudianos de la contratransferencia es una lectura enraizada en lo imaginario, una concepción de la cura basada en la relación de yo a yo. Ahora bien, Lacan nunca dejó de destacar el anudamiento de los tres registros -imaginario, simbólico y real-, y en este sentido consideramos que la contratransferencia tiene la riqueza de permitirnos pensar e interrogar esa articulación, y cómo se juega en la posición del analista. Consideramos que el recurso a la contratransferencia como posibilidad de pensar la posición del analista en el registro de lo imaginario resulta una referencia importante y necesaria, ya que si bien su yo tiene que "jugar al muerto", nunca puede ser abolido de manera permanente. Lo que introduce en este punto el deseo del analista es la posibilidad de plantear una función que permite que la contratransferencia, con sus implicancias en lo imaginario, no sea la que comande la cura.

En la tesis se sostiene que dar lugar a la contratransferencia en la reflexión teórico-clínica no implica perder de vista 
los peligros y excesos que puede conllevar en caso de hacer de ella el eje de la dirección de la cura y perder de vista la singularidad del paciente. De esta forma se propone, como manera de salir de la encerrona que implica la aparente oposición y relación de exclusión o sustitución entre la contratransferencia y el deseo del analista, la apelación a la categoría de "escucha analítica”. En el decir de Fernández Miranda "el reconocimiento de la irreductible alteridad del paciente, transforma la contratransferencia en escucha analítica" (Fernández Miranda, 2016).

Sin perder de vista las inconsistencias y las contradicciones que condensa el término, consideramos importante sostener el valor de la contratransferencia como el nombre de la necesaria implicación del analista en la transferencia, con los efectos imaginarios que esto puede implicar, entendiendo que su sustitución por la noción de "deseo del analista" corre el peligro de llevarnos a una concepción demasiado uniforme y "aconflictual" (Guyomard, 2011: 11) de la posición del analista. Se destaca que hay algo en la "impropiedad" misma del término, en su carácter problemático, en sus movimientos y contradicciones internas, que invita a la reflexión y a la interrogación teórico-clínica -particularmente respecto del lugar del analista- como no lo hacen otros conceptos psicoanalíticos como el de transferencia y deseo del analista.

Finalmente, la tesis deja abiertos interrogantes que inauguran otras líneas de investigación. En este punto se destaca la pregunta por la contratransferencia llevada más allá de lo teórico-clínico, al campo de lo político-institucional, gracias a la cual se podría interrogar, con nuevas herramientas, la cuestión de la puesta en primer plano, o por el contrario el borramiento, de la contratransferencia en los distintos ámbitos institucionales del psicoanálisis (particularmente la IPA y la AMP).

\section{REFERENCIAS BibLIOGRÁFICAS}

Adorno, T. (1962). "El ensayo como forma”. En Pensamiento de los confines, Número 5, octubre de 1998, 247-259. Buenos Aires: Fondo de Cultura Económica.

Baños, L. y Steinberg, I. (2012). Dificultades de la práctica del psicoanálisis. Rosario: Homo Sapiens.

Cabral, A. (2009). Lacan y el debate sobre la contratransferencia. Buenos Aires: Letra Viva.

Etchegoyen，H. (1986). "Contratransferencia: descubrimiento y redescubrimiento", "Contratransferencia y relación de objeto" y "Contratransferencia y proceso psicoanalítico", Los fundamentos de la técnica psicoanalitica. Buenos Aires: Amorrortu Editores, 236-270. 
Fernández Miranda, J. (2016). "La escucha y la transmisión. Apuntes para un pensamiento psicoanalítico". (Texto inédito).

Freud, S. (1905 [1901]) "Fragmento de análisis de un caso de histeria (Dora)", Sigmund Freud. Obras Completas. Buenos Aires: Amorrortu (2003), Volumen 7, 1-107.

(1909). “A propósito de un caso de neurosis obsesiva”, Sigmund Freud. Obras Completas. Buenos Aires: Amorrortu (2003), Volumen 10, 119-194.

(1910). "Las perspectivas futuras de la terapia psicoanalítica", Sigmund Freud. Obras Completas. Buenos Aires: Amorrortu (1999), Volumen 11, 129-142.

(1912). "Consejos al médico sobre el tratamiento psicoanalítico", Sigmund Freud. Obras Completas. Buenos Aires: Amorrortu (2001), Volumen 12, 107-119.

(1915 [1914]). "Puntualizaciones sobre el amor de transferencia”, Sigmund Freud. Obras Completas. Buenos Aires: Amorrortu (2001), Volumen 12, 159-174.

(1920). "Sobre la psicogénesis

de un caso de homosexualidad femenina", Sigmund Freud. Obras Completas. Buenos Aires: Amorrortu (2001), Volumen 18, 137-164.

(1933 [1932]). "Nuevas conferencias de introducción al psicoanálisis”, “30a conferencia. Sueño y ocultismo", Sigmund Freud. Obras Completas. Buenos Aires: Amorrortu (2001), Volumen 22, 29-52.

(1940 [1938]). "Esquema del psicoanálisis”, Sigmund Freud. Obras
Completas. Buenos Aires: Amorrortu (2001), Volumen 23, 133-209.

Geissmann, C. (2002). "Contratransferencia”. En AA.VV (2002). Diccionario internacional de Psicoanálisis. Conceptos, nociones, biografías, obras, acontecimientos, instituciones. Madrid: Ediciones Akal (2007), 300-301.

Gentile, A. (2009). "Conceptos psicoanalíticos en los fundamentos de la psicología". Proyecto de investigación 2009/12. Secretaría de Ciencia y Tecnología, Universidad Nacional de Rosario.

Ginzburg, C. (2004). "Huellas. Raíces de un paradigma indiciario", Tentativas. Rosario: Prohistoria ediciones, 93155.

Giordano, A. (1998). "La crítica de la crítica y el recurso al ensayo". En Actas del Sexto Congreso Internacional del CELCIRP "La figura del intelectual en la producción cultural rioplatense de fines del siglo XIX a fines del siglo $X X$ ”, 49-58. 25 al 27 de junio 1998, Nueva York, Fordham University, Lincoln Center Campus.

Guyomard, P. (2011). "Lacan et le contre-transfert: le contre-coup du transfert” y “Au dernier terme”. En Guyomard, P. et al. (2001) Lacan et le contre-transfert. Paris: PUF. 11-75. Citas traducidas por C. Greca.

Heimann, P. (1949-1950). "Sobre la contratransferencia", en Revista de Psicoanálisis, Asociación Psicoanalítica de Madrid. Número 32, mayo de 2000, 43-49. Madrid: Asociación Psicoanalítica de Madrid. 
LACAN, J. (1951). "Intervención sobre la transferencia", Escritos. Buenos Aires: Siglo XXI (2007), Volumen 1, 209 220.

(1958). "La dirección de la cura y los principios de su poder”, Escritos. Buenos Aires: Siglo XXI (2008), Volumen 2, 559-615.

(1960-1961). "La transferencia”, Lacan. El seminario. Buenos Aires: Paidós (2006), Volumen 8.

(1962-1963). "La Angustia", Lacan. El seminario. Buenos Aires: Paidós (2007), Volumen 10. (1964). "Los cuatro conceptos fundamentales del Psicoanálisis”, Lacan. El seminario. Buenos Aires: Paidós (2006), Volumen 11.

LefF, G. (2007). Juntos en la chimenea. La contratransferencia, las "mujeres analistas” y Lacan. México: Editorial Psicoanalítica de la Letra.

Little, M. (1951). “Contratransferencia y respuesta del paciente", Acheronta. Revista de Psicoanálisis y Cultura. Número 8, diciembre de 1998. URL: http://www.acheronta.org/acheronta8/little1.htm (última consulta: 08/12/15).

(1957). “ “ $R$ ” - La respuesta total del analista a las necesidades de su paciente". Traducción castellana: A. Gasparino. y J. Castelo, mayo-junio de 1997, para el Colectivo GRITA (Grupo de Investigación en Técnica de la Psicoterapia Psicoanalítica). URL: http://www. sauval.com/angustia/MLittleRT.pdf (última consulta: 07/12/15).

Miller, J.-A. (2003). "Contratransferencia e intersubjetividad", en $R e$ - vista Freudiana, número 38, año 2003. URL: http://es.scribd.com/ doc/23258313/Contratransferencia-e-Intersubjetividad\#scribd (última consulta: 13/03/2016).

Money-Kyrle, R. (1956). “Contratransferencia normal y algunas de sus desviaciones", en Revista uruguaya de Psicoanálisis, Asociación Psicoanalítica de Uruguay. Tomo IV, número 1, 1961-1962, 150-163. Montevideo: Asociación Psicoanalítica de Uruguay.

RABANT, C. (1992). "Sobre la interpretación en general”. En Inventar lo real. La desestimación entre perversión y psicosis. Buenos Aires: Ediciones Nueva Visión (1993), 15-26.

Ritvo, J. B. (1997). “Afectos, síntoma, fantasma”, en Ritvo, J. B. y Kuri, C. (1997). Ensayo de las Razones. Acto y Argumentación en Psicoanálisis. Buenos Aires: Letra Viva Ediciones, 65-93.

Tower, L. (1955). "La contratransferencia”, en Me cayó el veinte. Revista de psicoanálisis. Número 3: "Abajarse, o no”, primavera de 2001, 115-139. México: Epeele.

Weissmann, J. C. (1994). “Contratransferencia: su origen”, en Revista de Psicoanálisis, Asociación Psicoanalítica Argentina. Tomo LI, número 3, mayo-julio de 1994, 561-586. Buenos Aires: Asociación Psicoanalítica Argentina.

WidlöCher, D., Miller, J.-A. y Granger, B. (Coord.) (2002). "El porvenir del psicoanálisis", en Revista de Psicoanálisis, Asociación Psicoanalítica Argentina. Tomo LX, número 4, oc- 
tubre-diciembre de 2003, 1051-1070. Buenos Aires: Asociación Psicoanalítica Argentina.

WIDLÖCHER, D. (2011). "Le contretransfert, un autre transfert". En Guyomard, P. et al. (2001) Lacan et le contre-transfert. Paris: PUF. 93-112. Citas traducidas por C. Greca.

WinnicotT, D. (1960) "La contratransferencia". En Los procesos de maduración y el ambiente facilitador. Estudios para una teoría del desarrollo emocional (1965). Buenos Aires: Paidós (1993), 207-216.
RESEÑa CURRICULAR DEL AUTOR

Cecilia Greca es Psicóloga, Profesora en Psicología y Magíster en Psicoanálisis, graduada en la Facultad de Psicología de la Universidad Nacional de Rosario. Forma parte de la cátedra "Psicología" de la misma Facultad, en donde se desempeña como docente e investigadora. Trabaja como psicoanalista en el ámbito privado e integra el equipo del Dispositivo Integral de Abordaje Territorial (DIAT Rosario) como profesional dependiente del Ministerio de Desarrollo Social de la Provincia de Santa Fe. 